C. M. Ringel

Nagoya Math. J.

Vol. 183 (2006), 143-160

\title{
THE THEOREM OF BO CHEN AND HALL POLYNOMIALS
}

\author{
CLAUS MICHAEL RINGEL \\ Dedicated to George Lusztig on the occasion of \\ his sixtieth birthday
}

\begin{abstract}
Let $\Lambda$ be the path algebra of a Dynkin quiver. A recent result of Bo Chen asserts that $\operatorname{Hom}(X, Y / X)=0$ for any Gabriel-Roiter inclusion $X \subseteq Y$. The aim of the present note is to give an interpretation of this result in terms of Hall polynomials, and to extend it in this way to representationdirected split algebras. We further show its relevance when dealing with arbitrary representation-finite split algebras.
\end{abstract}

\section{$\S 1$. Introduction}

Let $k$ be a field. We call a $k$-algebra $\Lambda$ to be split provided the endomorphism ring of any simple $\Lambda$-module is just $k$. A finite-dimensional $k$-algebra $\Lambda$ is said to be representation-directed provided there are (up to isomorphism) only finitely many indecomposable $\Lambda$-modules, say $M_{1}, M_{2}, \ldots, M_{m}$, and these modules can be indexed in such a way that $\operatorname{Hom}\left(M_{i}, M_{j}\right)=0$ for $i>j$. The most important examples of representation-directed split $k$-algebras are the path algebras of the Dynkin quivers (of type $A_{n}, D_{n}, E_{6}$, $\left.E_{7}, E_{8}\right)$.

We will recall below the notion of a Gabriel-Roiter submodule of an indecomposable $\Lambda$-module $Y$ : such a submodule $X$ always exists in case $Y$ is not simple. Gabriel-Roiter submodules $X$ of $Y$ have many nice properties, in particular both $X$ and $Y / X$ are indecomposable.

Bo Chen Theorem. Let $\Lambda$ be a representation-directed split $k$-algebra. Let $Y$ be an indecomposable $\Lambda$-module and $X$ a Gabriel-Roiter submodule of $Y$. Then $\operatorname{Hom}(X, Y / X)=0$.

Received June 28, 2005.

Revised February 24, 2006.

2000 Mathematics Subject Classification: Primary 16G20, 16G60; Secondary 17B37, $81 \mathrm{R} 50$. 
Bo Chen $[\mathrm{C}]$ gave a proof in case $\Lambda$ is the path algebra of a Dynkin quiver. His proof is combinatorial and is based on a detailed study of the corresponding Auslander-Reiten quiver. Bo Chen conjectured that the statement should be true for any representation-directed algebra. The aim of the present note is to verify this conjecture using knowledge on Hall polynomials. The relationship exhibited in this way, between the GabrielRoiter measure and Hall theory, may be of further interest. In order to pass from finite fields to $\mathbb{Q}$, we use integral representations.

Let $\Lambda$ be a representation-directed split $k$-algebra. Then $\operatorname{End}(X)=k$ for any indecomposable $\Lambda$-module $X$. Also, for any exact sequence

$$
0 \longrightarrow X \longrightarrow Y \longrightarrow Z \longrightarrow 0
$$

of indecomposable $\Lambda$-modules $X, Y, Z$, one necessarily has $\operatorname{Hom}(Z, X)=0$. Thus, the assertion $\operatorname{Hom}(X, Z)=0$ means that the pair $X, Z$ is a pair of "orthogonal bricks" with $\operatorname{dim}_{k} \operatorname{Ext}^{1}(Z, X)=1$. Thus, the Bo Chen Theorem shows that there is an inductive procedure in order to construct all the indecomposables starting from the simple modules, namely forming extensions of orthogonal bricks. In the case when $\Lambda$ is hereditary, this procedure was studied by Schofield (see [S] and [R4]), and we propose to call the exact sequences with $X, Y, Z$ indecomposable and $\operatorname{Hom}(X, Z)=0$ Schofield sequences also for any representation-directed split $k$-algebra $\Lambda$. In the hereditary case one knows the precise number of pairs $X, Z$ which yield a Schofield sequence $(*)$, it is $n_{Y}-1$, where $n_{Y}$ is the number of isomorphism classes of composition factors of $Y$ (a corresponding assertion is not known in general). Whereas it is easy to construct $Y$ given $X$ and $Z$, there does not yet exist a convenient procedure to determine the possible modules $X$ (and then $Z$ ), when $Y$ is given. But the Bo Chen Theorem provides a method to find at least some of these modules $X$, namely the Gabriel-Roiter submodules of $Y$.

In the last section we show the relevance of the Bo Chen Theorem when dealing with arbitrary representation-finite split $k$-algebras. The covering theory of Bongartz-Gabriel [BG] allows to reduce questions concerning representation-finite algebras to representation-directed algebras. Thus, consider a representation-finite split $k$-algebra $\Lambda$ and an indecomposable $\Lambda$ module $Y$. In general, there will be no orthogonal pairs of $\Lambda$-modules, as the case of $\Lambda$ being a local algebra shows. However, if we take a GabrielRoiter submodule $X$ of $Y$ and write $Z=Y / X$, then such pairs $X, Z$ may 
be considered as a sort of substitute, since they give rise to orthogonal pairs for a corresponding covering algebra.

\section{$\S 2 . \quad$ Gabriel-Roiter inclusions}

Let $\mathcal{C}$ be a length category (i.e. an abelian category, such that any object has finite length). For any object $X$ in $\mathcal{C}$, we denote its length by $|X|$. The Gabriel-Roiter measure of an object of $\mathcal{C}$ is an invariant which takes values in a countable totally ordered set. It has been introduced by Gabriel [G] (under the name of "Roiter measure") in order to focus the attention to the induction process used by Roiter in his proof of the first Brauer-Thrall conjecture. Note that any countable totally ordered set may be considered as a subset of the rational numbers $\mathbb{Q}$, thus we may (and will) assume that the Gabriel-Roiter measure $\mu(X)$ of any object $X$ in $\mathcal{C}$ is a rational number. Indeed, one may reformulate Gabriel's definition as follows (see [R6], [R7]): Let $\mu(0)=0$ for the zero object 0 . Given a non-zero object $X$, we may assume by induction that $\mu\left(X^{\prime}\right)$ is already defined for any proper subobject $X^{\prime}$ of $X$. Let

$$
\mu(X)=\max \mu\left(X^{\prime}\right)+\left\{\begin{array}{cc}
2^{-|X|} \\
0
\end{array} \text { in case } X \text { is } \quad\right. \text { indecomposable, }
$$

with the maximum being taken over all proper subobjects $X^{\prime}$ of $X$. Note that the maximum exists, since one observes (along the induction) that $\mu(X)=\sum_{i \in I(X)} 2^{-i}$ for a set $I(X) \subseteq\{1,2, \ldots,|X|\}$. The original definition by Gabriel $[\mathrm{G}]$ used the set $I(X)$ itself as the Gabriel-Roiter measure of $X$, with a corresponding totally ordering on the set of finite subsets of natural numbers.

If $Y$ is an indecomposable object of length $n$ which is not simple, then there exists an indecomposable subobject $X$ of $Y$ such that $\mu(Y)=\mu(X)+$ $2^{-n}$, which will be called a Gabriel-Roiter subobject of $Y$. The GabrielRoiter subobjects $X$ may also be characterized as follows: first, $X$ is an indecomposable subobject of $Y$; second, $\mu(X)<\mu(Y)$; third, for any proper subobject $Y^{\prime}$ of $Y$, we have $\mu\left(Y^{\prime}\right) \leq \mu(X)$. Note that $Y$ may contain several Gabriel-Roiter subobjects. They are not unique, not even unique up to isomorphism, but all have the same length. It has been shown in [R6] that for any Gabriel-Roiter subobject $X$ of $Y$, the factor object $Y / X$ is indecomposable. Note that if $X$ is a Gabriel-Roiter subobject of $Y$ and $f: X \rightarrow Y$ is any monomorphism, then also $f(X)$ is a Gabriel-Roiter 
subobject of $Y$, and thus the cokernel of any such $f$ is indecomposable. On the other hand, let us consider now the set $\operatorname{Sing}(X, Y)$ of maps $f: X \rightarrow Y$ which are not monomorphisms.

Proposition 1. Let $Y$ be indecomposable and not simple, let $X$ be a Gabriel-Roiter subobject of $Y$. Then $\operatorname{Sing}(X, Y)$ is closed under addition.

Proof. For $i=1,2$, let $f_{i}: X \rightarrow Y$ be maps such that $f_{1}+f_{2}$ is a monomorphism. We want to show that at least one of $f_{1}, f_{2}$ is a monomorphism. Let $Y_{i}$ be the image of $f_{i}$, for $i=1,2$. Since $\left|Y_{i}\right| \leq|X|<|Y|$, we see that $Y_{i}$ is a proper subobject of $Y$, thus $\mu\left(Y_{i}\right) \leq \mu(X)$. As a consequence, $\max \left(\mu\left(Y_{1}\right), \mu\left(Y_{2}\right)\right) \leq \mu(X)$.

Since $f_{1}+f_{2}$ is a monomorphism, the map

$$
\left[\begin{array}{l}
f_{1} \\
f_{2}
\end{array}\right]: X \longrightarrow Y_{1} \oplus Y_{2}
$$

is a monomorphism too, since its composition with the summation map: $Y_{1} \oplus Y_{2} \rightarrow Y$ is just $f_{1}+f_{2}$. Using Gabriel's main property [R6], it follows from

$$
\max \left(\mu\left(Y_{1}\right), \mu\left(Y_{2}\right)\right) \leq \mu(X),
$$

that the mapping $\left[\begin{array}{l}f_{1} \\ f_{2}\end{array}\right]$ is a split monomorphism. But this is only possible in case at least one of the maps $f_{i}: X \rightarrow Y_{i}$ is a split monomorphism. In particular this $f_{i}$ is a monomorphism. (Another proof will be given in [R7].)

Here is a consequence in case $\Lambda$ is a representation-finite $k$-algebra. Assume that $M_{1}, \ldots, M_{m}$ are all the indecomposable $\Lambda$-modules, one from each isomorphism class. For every pair of modules $M_{i}, M_{j}$ choose irreducible maps $f_{j i}^{r}: M_{i} \rightarrow M_{j}$, say $1 \leq r \leq m_{i j}$, such that the residue classes modulo the square of the radical of $\bmod \Lambda$ form a $k$-basis of the bimodule $\operatorname{Irr}\left(M_{i}, M_{j}\right)$ of irreducible maps. Let $\mathcal{B}$ be the set of these maps $f_{j i}^{r}$ for all the $i, j, r$. One may call $\mathcal{B}$ a basis of irreducible maps for $\bmod \Lambda$. Then it is well-known (and not difficult to see) that any map $M_{a} \rightarrow M_{b}$ is a linear combination of compositions of maps from $\mathcal{B}$. Proposition 1 implies the following: If $M_{a}$ is a Gabriel-Roiter submodule of $M_{b}$, then there is a monomorphism $M_{a} \rightarrow M_{b}$ which is a composition of maps in $\mathcal{B}$.

Proof. If all compositions of maps in $\mathcal{B}$ would belong to $\operatorname{Sing}\left(M_{a}, M_{b}\right)$, then $\operatorname{Sing}\left(M_{a}, M_{b}\right)=\operatorname{Hom}\left(M_{a}, M_{b}\right)$. But by assumption, there exists an inclusion $M_{a} \rightarrow M_{b}$. 


\section{$\S 3$. Counting submodules}

Now assume that $k$ is a finite field, say with $q$ elements. Let $\Lambda$ be a finite-dimensional $k$-algebra. Let $X, Y$ be indecomposable finite-dimensional $\Lambda$-modules. Note that $\operatorname{Sing}(X, Y)$ is not only a subgroup, but a $k$-subspace of $\operatorname{Hom}(X, Y)$.

Proposition 2. Assume that $X$ is a Gabriel-Roiter submodule of $Y$. Let

$$
\begin{array}{ll}
e=\operatorname{dim}_{k} \operatorname{End}(X), & r=\operatorname{dim}_{k} \operatorname{rad} \operatorname{End}(X), \\
h=\operatorname{dim}_{k} \operatorname{Hom}(X, Y), & s=\operatorname{dim}_{k} \operatorname{Sing}(X, Y) .
\end{array}
$$

Then the number $u_{X}^{Y}$ of submodules of $Y$ which are isomorphic to $X$ is

$$
q^{s-r}\left(q^{h-s}-1\right)\left(q^{e-r}-1\right)^{-1}
$$

(and $h>s \geq r, e>r)$.

Proof. The inequality $r<e$ is obvious. Also, $\operatorname{Sing}(X, Y)$ is a proper subset of $\operatorname{Hom}(X, Y)$, since there is the inclusion map $X \subset Y$, thus $s<h$. The set $\operatorname{Hom}(X, Y) \backslash \operatorname{Sing}(X, Y)$ is the set of monomorphisms $X \rightarrow Y$, thus the number of monomorphisms $X \rightarrow Y$ is $q^{h}-q^{s}$. Two monomorphisms $f, f^{\prime}: X \rightarrow Y$ have the same image if and only if there exists an automorphism $g: X \rightarrow X$ with $f^{\prime}=f g$, and such an automorphism is unique: The group $\operatorname{Aut}(X)$ of automorphisms of $X$ operates freely on $\operatorname{Hom}(X, Y) \backslash \operatorname{Sing}(X, Y)$, thus we see that

$$
|\operatorname{Hom}(X, Y) \backslash \operatorname{Sing}(X, Y)|=|\operatorname{Aut}(X)| \cdot u_{X}^{Y}
$$

Of course, $\operatorname{Aut}(X)=\operatorname{End}(X) \backslash \operatorname{rad} \operatorname{End}(X)$, thus $|\operatorname{Aut}(X)|=q^{e}-q^{r}$ and therefore

$$
q^{h}-q^{s}=\left(q^{e}-q^{r}\right) \cdot u_{X}^{Y}
$$

Since $q^{r}$ divides the right side, it devides the left side $q^{h}-q^{s}=q^{s}\left(q^{h-s}-1\right)$, thus $q^{r}$ divides $q^{s}$ and therefore $r \leq s$.

\section{§4. Hall polynomials for representation-directed algebras}

We return now to a representation-directed split $k$-algebra $\Lambda$. First we deal with an arbitrary field $k$. Let $X$ be a Gabriel-Roiter submodule of the $\Lambda$-module $Y$, and let $Z=Y / X$. We have mentioned above that $Z$ is an indecomposable $\Lambda$-module. 
Let $K_{0}(\Lambda)$ be the Grothendieck group of mod $\Lambda$ (of all finite length modules modulo exact sequences). Given a finite length module $M$, we denote by $\operatorname{dim} M$ the corresponding element in $K_{0}(\Lambda)$. If $M$ is an indecomposable $\Lambda$-module, then (see [R1]) we know that $\operatorname{End}(M)=k, \operatorname{Ext}^{1}(M, M)=0$ and that the isomorphism class of $M$ is uniquely determined by $\operatorname{dim} M$. We may fix a representative in the isomorphism class of $M$ and denote it by $M(\mathbf{x})$ or $M(\mathbf{x}, k)$, where $\mathbf{x}=\operatorname{dim} M$. Alternatively, we may consider $\mathbf{x}$ as a vertex of the Auslander-Reiten quiver $\Gamma$ of $\Lambda$ so that $M(\mathbf{x})$ is an indecomposable $\Lambda$-module belonging to the isomorphism class $\mathbf{x}$.

Let us assume that $k$ is a finite field. Given any embedding $f: X \rightarrow$ $Y$, the cokernel of $f$ is indecomposable, as we have noted above, and its dimension vector is $\operatorname{dim} Y-\operatorname{dim} X=\operatorname{dim} Z$. But $Z$ is up to isomorphism the only indecomposable module with this dimension vector. Thus the cokernel of $f$ is isomorphic to $Z$. If we denote by $F_{Z X}^{Y}$ the number of submodules $U$ of $Y$ isomorphic to $X$ with $Y / U$ isomorphic to $Z$, then this is just the number of submodules of $Y$ isomorphic to $X$. Let $\mathbf{x}=\operatorname{dim} X, \mathbf{y}=$ $\operatorname{dim} Y, \mathbf{z}=\operatorname{dim} Z$. It has been shown in [R2] that $h=\operatorname{dim}_{k} \operatorname{Hom}(X, Y)$ only depends on $\mathbf{x}, \mathbf{y}$. For $e=\operatorname{dim}_{k} \operatorname{End}(X)$ we even know that $e=1$, since $\Lambda$ is assumed to be representation-directed and split, and consequently $r=$ $\operatorname{dim}_{k} \operatorname{rad} \operatorname{End}(S)=0$. But we do not yet know that $s=\operatorname{dim}_{k} \operatorname{Sing}(X, Y)$ depends only on $\mathbf{x}$ and $\mathbf{y}$.

In order to overcome this difficulty, observe that for a field extension $k \subset k^{\prime}$ and the scalar extension $\Lambda^{k^{\prime}}=\Lambda \otimes_{k} k^{\prime}, X^{k^{\prime}}=X \otimes_{k} k^{\prime}, Y^{k^{\prime}}=Y \otimes_{k} k^{\prime}$, we have an inclusion

$$
\operatorname{Sing}_{\Lambda}(X, Y) \otimes_{k} k^{\prime} \subseteq \operatorname{Sing}_{\Lambda^{k^{\prime}}}\left(X^{k^{\prime}}, Y^{k^{\prime}}\right) \subseteq \operatorname{Hom}_{\Lambda^{k^{\prime}}}\left(X^{k^{\prime}}, Y^{k^{\prime}}\right) .
$$

Thus, if we set $s\left(k^{\prime}\right)=\operatorname{dim}_{k^{\prime}} \operatorname{Sing}_{\Lambda^{k^{\prime}}}\left(X^{k^{\prime}}, Y^{k^{\prime}}\right)$, then this function $s$ is monotone with respect to field extensions and bounded by $\operatorname{dim}_{k} \operatorname{Hom}(X, Y)$, thus it will stabilize for large finite fields; let this value be $s$. It follows that the polynomial

$$
\psi_{\mathbf{z x}}^{\mathbf{y}}(T)=\left(T^{h}-T^{s}\right)(T-1)^{-1}=T^{s}\left(T^{h-s-1}+\cdots+T+1\right),
$$

has the evaluation

$$
\psi_{\mathbf{z x}}^{\mathbf{y}}(q)=F_{M(\mathbf{z}, k), M(\mathbf{x}, k)}^{M(\mathbf{y}, k)},
$$

for large finite fields $k$ with $|k|=q$. But this means that $\psi_{\mathbf{z x}}^{\mathbf{y}}(T)$ coincides with the Hall polynomial $\phi_{\mathbf{z} \mathbf{x}}^{\mathbf{y}}(T)$ which satisfies

$$
\phi_{\mathbf{z x}}^{\mathbf{y}}(q)=F_{M(\mathbf{z}, k), M(\mathbf{x}, k)}^{M(\mathbf{y}, k)},
$$


for all finite fields $k$.

In [R3], all the Hall polynomials $\phi_{\mathbf{z x}}^{\mathbf{y}}(T)$ for representation-directed algebras and the dimension vectors $\mathbf{x}=\operatorname{dim} X, \mathbf{y}=\operatorname{dim} Y, \mathbf{z}=\operatorname{dim} Z$ of indecomposable modules $X, Y, Z$ have been calculated: here is the corresponding list for the split algebras:

$$
\begin{gathered}
0, \quad 1, \quad T-2, \quad(T-2)^{2}, \quad(T-2)^{3}, \\
T^{3}-5 T^{2}+10 T-7, \quad(T-2)\left(T^{3}-4 T^{2}+8 T-6\right), \\
T^{5}-6 T^{4}+15 T^{3}-23 T^{2}+25 T-13 .
\end{gathered}
$$

It follows that we must have $\phi_{\mathbf{z x}}^{\mathbf{y}}=1$, and therefore $s=0$ and $h-s-1=0$, thus $h=1$. But $\operatorname{Hom}(X, Y)=k$ immediately implies that $\operatorname{Hom}(X, Z)=0$. Just look at the long exact sequence for $0 \rightarrow X \rightarrow Y \rightarrow Z \rightarrow 0$, and note that $\operatorname{Hom}(X, X)=k$ and $\operatorname{Ext}^{1}(X, X)=0$. (Let us add also the following remark: since $s=0$ and $s(k) \leq s$ for all finite fields $k$, we see that always $s(k)=0$.)

This furnishes a proof for the Bo Chen Theorem in case the base field $k$ is finite.

Remark 1. The Hall polynomials $\phi_{\mathbf{z x}}^{\mathbf{y}}(q)$ with $\mathbf{x}, \mathbf{y}, \mathbf{z}$ dimension vectors of indecomposable modules have been calculated in order to determine the structure of the Hall algebras $\mathcal{H}(\Lambda)$. This was done in analogy to the case of the classical Hall algebras $\mathcal{H}(R)$, where $R$ is a discrete valuation ring (say $R=\mathbb{Z}_{(p)}$ or $R=k[[T]]$ ), as studied by Steinitz and Philip Hall (see $[\mathrm{M}])$. Whereas in the classical case the only Hall polynomials $\phi_{\mathbf{z x}}^{\mathbf{y}}(T)$, with $\mathbf{x}, \mathbf{y}, \mathbf{z}$ referring to indecomposable $R$-modules, are the polynomials 0 and 1 , it was quite a surprise to see that for $\Lambda$ representation-directed, polynomials of degree up to 5 can occur. The numerous investigations of Hall polynomials in the classical case are devoted to deal with decomposable (not indecomposable) $R$-modules. The corresponding simple nature of the Hall polynomials for Gabriel-Roiter inclusions for representation-directed split algebras could be of interest for the determination of further Hall polynomials.

Remark 2. We have mentioned in the introduction that the exact sequences

$$
0 \longrightarrow X \longrightarrow Y \longrightarrow Z \longrightarrow 0
$$


with $X$ a Gabriel-Roiter submodule of $Y$ are special cases of Schofield sequences. In terms of the Hall multiplication (see [R2], [L]) we see the following: such a Schofield sequence $(*)$ yields that the isomorphism class $[Y]$ can be written in the corresponding Hall algebra as the commutator $[[Z],[X]]$, since

$$
[Z] \diamond[X]=[Y]+[X \oplus Z], \quad[X] \diamond[Z]=[X \oplus Z]
$$

where $\diamond$ denotes the multiplication in the (untwisted) Hall algebra $\mathcal{H}(\Lambda)$.

\section{§5. Reduction to finite fields}

In the last section, we gave a proof of the Bo Chen Theorem for $k$ a finite field. One may use this special case in order to obtain a proof in general. If $k$ is any field, we denote by $k_{0}$ its prime field.

First we note the following:

(1) If there exists a Gabriel-Roiter inclusion $M(\mathbf{x}, k) \rightarrow M(\mathbf{y}, k)$ for some finite field $k$, then there is an embedding $M\left(\mathbf{x}, k^{\prime}\right) \rightarrow M\left(\mathbf{y}, k^{\prime}\right)$ for any field $k^{\prime}$.

Proof. This is an immediate consequence of the Bo Chen Theorem, since the existence of a Schofield sequence $0 \rightarrow M(\mathbf{x}, k) \rightarrow M(\mathbf{y}, k) \rightarrow$ $M(\mathbf{z}, k) \rightarrow 0$ is independent of $k$.

Since we are looking only at an indecomposable module $Y$ and a submodule $X$, we may assume that $Y$ is faithful. A representation-directed split algebra $\Lambda$ with a faithful indecomposable module is a tilted algebra and therefore $\Lambda$, as well as all the indecomposable $\Lambda$-modules, are defined over the prime field $k_{0}$ of $k$. (As a tilted algebra, $\Lambda$ is the endomorphism ring $\operatorname{End}_{H}(T)$, where $H$ is a finite-dimensional hereditary $k$-algebra, and $T$ is a tilting $H$-module. With $\Lambda$ also $H$ is a split $k$-algebra [R5], thus $H=k Q$ is the path algebra of a finite quiver $Q$ without oriented cycles. But then $H=\left(k_{0} Q\right)^{k}$. Also, there is a tilting $k_{0} Q$-module $T_{0}$ with $T=\left(T_{0}\right)^{k}$. If $\Lambda_{0}=\operatorname{End}_{k_{0} Q}\left(T_{0}\right)$, then $\Lambda=\left(\Lambda_{0}\right)^{k}$.)

(2) If there exists a Gabriel-Roiter inclusion $M(\mathbf{x}, k) \rightarrow M(\mathbf{y}, k)$ for some field $k$, then there is an embedding $M\left(\mathbf{x}, k_{0}\right) \rightarrow M\left(\mathbf{y}, k_{0}\right)$. 
Proof. Choose a basis $\mathcal{B}$ of irreducible maps for $\bmod \Lambda$. Since $\Lambda$ is defined over the prime field $k_{0}$, we can assume that all the elements of $\mathcal{B}$ are defined over the prime field. As we have mentioned at the end of Section 2, there is a Gabriel-Roiter inclusion $M(\mathbf{x}, k) \rightarrow M(\mathbf{y}, k)$ which is a composition of elements of $\mathcal{B}$. This composition yields a map $M\left(\mathbf{x}, k_{0}\right) \rightarrow$ $M\left(\mathbf{y}, k_{0}\right)$ which has to be a monomorphism.

(3) If there exists a Gabriel-Roiter inclusion $M(\mathbf{x}, \mathbb{Q}) \rightarrow M(\mathbf{y}, \mathbb{Q})$, then there is an embedding $M\left(\mathbf{x}, \mathbb{F}_{p}\right) \rightarrow M\left(\mathbf{y}, \mathbb{F}_{p}\right)$ for almost all prime numbers $p$.

Proof. We will use integral representations as considered (for quivers) by Crawley-Boevey [CB]. Before we do this, let us recall the knitting procedure for a representation-directed split $k$-algebra $\Lambda$ : One may construct the indecomposable $\Lambda$-modules $M_{1}, \ldots, M_{m}$ as well as a basis $\mathcal{B}$ of irreducible maps inductively as follows: Assume that $M_{1}, \ldots, M_{i-1}$ and the irreducible maps between them are already constructed. If $M_{i}$ is projective, then $M_{i}=k \oplus \bigoplus_{j=1}^{r} N_{j}$ with modules $N_{j} \simeq M_{\sigma(j)}$, where $\sigma:\{1, \ldots, r\} \rightarrow\{1, \ldots, i-1\}$ is an injective map. Thus, up to isomorphism, one may assume that $\operatorname{rad} M_{i}=\bigoplus_{j=1}^{r} M_{\sigma(j)}$ and one adds the inclusion maps $M_{\sigma(j)} \rightarrow M_{i}$ to $\mathcal{B}$. If $M_{i}$ is not projective, then there is an Auslander-Reiten sequence

$$
0 \longrightarrow N_{0} \stackrel{\left(f_{j}\right)_{j}}{\longrightarrow} \bigoplus_{j=1}^{r} N_{j} \stackrel{\left(g_{j}\right)_{j}}{\longrightarrow} M_{i} \longrightarrow 0
$$

with modules $N_{j} \simeq M_{\sigma(j)}$, where this time $\sigma:\{0,1, \ldots, r\} \rightarrow\{1, \ldots, i-1\}$ is an injective map. Again, up to isomorphism, we can assume that we deal with an exact sequence of the form

$$
0 \longrightarrow M_{\sigma(0)} \stackrel{\left(f_{j}\right)_{j}}{\longrightarrow} \bigoplus_{j=1}^{r} M_{\sigma(j)} \stackrel{\left(g_{j}\right)_{j}}{\longrightarrow} M_{i} \longrightarrow 0,
$$

where the maps $f_{j}$ already belong to $\mathcal{B}$. In this way, the cokernel $M_{i}$ and the new irreducible maps $g_{j}$ are constructed using given modules and given maps. Note that in this way we reconstruct also the $k$-algebra $\Lambda$ itself (as the opposite of the endomorphsim ring of the direct sum of the indecomposable projective objects).

If we would use the same inductive procedure in order to construct integral representations, we would face the problem that starting with representations which use finitely generated free abelian groups, the corresponding 
cokernel may no longer be a free abelian group. But we can use the dual procedure, knitting the Auslander-Reiten quiver from right to left. Thus, assume that the $\Lambda$-modules $M_{i+1}, \ldots, M_{m}$ are already constructed. In case $M_{i}$ is injective, we can assume

$$
M_{i}=k \oplus \bigoplus_{j=1}^{r} M_{\sigma(j)}
$$

with an injective map $\sigma:\{1, \ldots, r\} \rightarrow\{i+1, \ldots, m\}$, whereas if $M_{i}$ is not injective, then we deal with an exact sequence

$$
0 \longrightarrow M_{i} \stackrel{\left(f_{j}\right)_{j}}{\longrightarrow} \bigoplus_{j=1}^{r} M_{\sigma(j)} \stackrel{\left(g_{j}\right)_{j}}{\longrightarrow} M_{\sigma(0)} \longrightarrow 0
$$

where now the maps $g_{j}$ are irreducible maps in $\mathcal{B}$ which already are constructed, and $\sigma:\{0,1, \ldots, r\} \rightarrow\{i+1, \ldots, m\}$ is an injective map. Again, we reconstruct the $k$-algebra $\Lambda$ itself (now as the opposite of the endomorphsim ring of the direct sum of the indecomposable injective objects).

Do the same with $k$ replaced by $\mathbb{Z}$. To be precise: Consider a representation-directed split $\mathbb{Q}$-algebra $\Lambda$ and let $\Gamma$ be its Auslander-Reiten quiver. The knitting procedure from right to left produces "integral representations" $M_{i}^{\mathbb{Z}}$ which are finitely generated free as abelian groups as well as a set $\mathcal{B}^{\mathbb{Z}}$ of maps between these objects, following the shape of $\Gamma$. We obtain at the same time a $\mathbb{Z}$-order $\Lambda^{\mathbb{Z}}$ in $\Lambda$, and the objects $M_{i}^{\mathbb{Z}}$ are indeed $\Lambda^{\mathbb{Z}}$ modules, and the maps in $\mathcal{B}^{\mathbb{Z}}$ are $\Lambda^{\mathbb{Z}}$-homomorphisms. Note that for the injective modules $M_{i}$, the corresponding integral representation is

$$
M_{i}^{\mathbb{Z}}=\mathbb{Z} \oplus \bigoplus_{j=1}^{r} M_{\sigma(j)}^{\mathbb{Z}}
$$

whereas for $M_{i}$ not injective, we get an exact sequence

$$
0 \longrightarrow M_{i}^{\mathbb{Z}} \stackrel{\left(f_{j}\right)_{j}}{\longrightarrow} \bigoplus_{j=1}^{r} M_{\sigma(j)}^{\mathbb{Z}} \stackrel{\left(g_{j}\right)_{j}}{\longrightarrow} M_{\sigma(0)}^{\mathbb{Z}} \longrightarrow 0
$$

starting with the maps $g_{j}$ already constructed in $\mathcal{B}^{\mathbb{Z}}$ and forming the kernel $M_{i}^{\mathbb{Z}}$. Note that $M_{i}^{\mathbb{Z}}$ considered as an abelian group is finitely generated and free, since it is constructed as a subgroup of a finitely generated free abelian group. In order to see that $g=\left(g_{j}\right)_{j}$ is surjective, we first note that 
cokernel of $g$ will be torsion, since $g \otimes_{\mathbb{Z}} \mathbb{Q}$ is surjective. But we also know that $g \otimes_{\mathbb{Z}} \mathbb{F}_{p}$ is surjective, for any $p$, thus the cokernel of $g$ has to be zero. The sequence splits as a sequence of abelian groups (since $M_{\sigma(0)}^{\mathbb{Z}}$ is free). Tensoring the sequence with any field $k$, one obtains an Auslander-Reiten sequences.

Altogether we see that for any $\mathbf{x} \in K_{0}(\Lambda)$, there exists an integral representation $M(\mathbf{x}, \mathbb{Z})$ such that

$$
M(\mathbf{x}, \mathbb{Z}) \otimes_{\mathbb{Z}} k=M(\mathbf{x}, k)
$$

for any field $k$. Also, in case there is an irreducible map $M(\mathbf{x}, k) \rightarrow M(\mathbf{y}, k)$, there is a corresponding map

$$
f: M(\mathbf{x}, \mathbb{Z}) \longrightarrow M(\mathbf{y}, \mathbb{Z})
$$

in $\mathcal{B}^{\mathbb{Z}}$ and

$$
f \otimes_{\mathbb{Z}} k: M(\mathbf{x}, k) \longrightarrow M(\mathbf{y}, k)
$$

is irreducible, for all fields $k$.

Now we can present the proof of (3). Consider a Gabriel-Roiter inclusion $M(\mathbf{x}, \mathbb{Q}) \rightarrow M(\mathbf{y}, \mathbb{Q})$. As we know from Section 2 , there is an embedding of $M(\mathbf{x}, \mathbb{Q})$ into $M(\mathbf{y}, \mathbb{Q})$ which is a composition of irreducible maps. Thus we see that there is a map

$$
u: M(\mathbf{x}, \mathbb{Z}) \longrightarrow M(\mathbf{y}, \mathbb{Z})
$$

which is a composition of maps in $\mathcal{B}^{\mathbb{Z}}$ and such that $u \otimes_{\mathbb{Z}} \mathbb{Q}$ is injective. But then $u \otimes_{\mathbb{Z}} \mathbb{F}_{p}$ is injective for almost all primes $p$ and this is an embedding

$$
u \otimes_{\mathbb{Z}} \mathbb{F}_{p}: M\left(\mathbf{x}, \mathbb{F}_{p}\right) \longrightarrow M\left(\mathbf{y}, \mathbb{F}_{p}\right),
$$

as required.

The assertions (1), (2), (3) have the following consequences:

$\left(\mathbf{1}^{\prime}\right)$ If $k$ is a finite field, and $k^{\prime}$ an arbitrary field, then $\mu(M(\mathbf{x}, k)) \leq$ $\mu\left(M\left(\mathbf{x}, k^{\prime}\right)\right)$.

Namely, if $I(M(\mathbf{x}, k))=\left\{n_{1}<n_{2}<\cdots<n_{t}\right\}$, then there is a sequence of Gabriel-Roiter inclusions

$$
M\left(\mathbf{x}_{1}, k\right) \longrightarrow M\left(\mathbf{x}_{2}, k\right) \longrightarrow \cdots \longrightarrow M\left(\mathbf{x}_{t}, k\right)=M(\mathbf{x}, k)
$$


with $\left|M\left(\mathbf{x}_{i}, k\right)\right|=n_{i}$. According to (1), there is a chain of inclusion maps

$$
M\left(\mathbf{x}_{1}, k^{\prime}\right) \longrightarrow M\left(\mathbf{x}_{2}, k^{\prime}\right) \longrightarrow \cdots \longrightarrow M\left(\mathbf{x}_{t}, k^{\prime}\right)=M\left(\mathbf{x}, k^{\prime}\right)
$$

and $\left|M\left(\mathbf{x}_{i}, k^{\prime}\right)\right|=n_{i}$. But this implies that $\mu(M(\mathbf{x}, k)) \leq \mu\left(M\left(\mathbf{x}, k^{\prime}\right)\right)$.

Similarly, (2) and (3) yield:

$\left.\mathbf{( 2}^{\prime}\right)$ If $k$ is an arbitrary field, then $\mu(M(\mathbf{x}, k)) \leq \mu\left(M\left(\mathbf{x}, k_{0}\right)\right)$.

$\left(\mathbf{3}^{\prime}\right)$ If $p \gg 0$, then $\mu(M(\mathbf{x}, \mathbb{Q})) \leq \mu\left(M\left(\mathbf{x}, \mathbb{F}_{p}\right)\right)$.

Combining these considerations, we see:

(4) For arbitrary fields $k, k^{\prime}$, we have:

$$
\mu(M(\mathbf{x}, k))=\mu\left(M\left(\mathbf{x}, k^{\prime}\right)\right) .
$$

Proof. If follows from $\left(\mathbf{1}^{\prime}\right)$ that $\mu(M(\mathbf{x}, k))=\mu\left(M\left(\mathbf{x}, k^{\prime}\right)\right)$ for arbitrary finite fields $k, k^{\prime}$. The combination of $\left(\mathbf{1}^{\prime}\right)$ and $\left(\mathbf{2}^{\prime}\right)$ shows the same for $k$ a field of characteristic $p>0$ and $k^{\prime}=k_{0}$ its prime field. Using in addition also $\left(\mathbf{3}^{\prime}\right)$, we see that the conclusion also holds for fields of characteristic 0 .

Proposition 3. Let $\Lambda$ be a representation-directed split $k$-algebra. The existence of a Gabriel-Roiter inclusion $X \rightarrow Y$ only depends on the position of $X$ and $Y$ in the Auslander-Reiten quiver of $\Lambda$ (and not on $k$ ).

Proof. According to (4), the Gabriel-Roiter measure of any $M(\mathbf{x}, k)$ does not depend on $k$. Thus, the assertions (1), (2) and (3) can be strengthened as follows:

$\left.\mathbf{( 1}^{\prime \prime}\right)$ If there is a Gabriel-Roiter inclusion $M(\mathbf{x}, k) \rightarrow M(\mathbf{y}, k)$ for some finite field $k$, then there is a Gabriel-Roiter inclusion $M\left(\mathbf{x}, k^{\prime}\right) \rightarrow M\left(\mathbf{y}, k^{\prime}\right)$ for any field $k^{\prime}$.

$\left(\mathbf{2}^{\prime \prime}\right)$ If there is a Gabriel-Roiter inclusion $M(\mathbf{x}, k) \rightarrow M(\mathbf{y}, k)$ for some field $k$, then there is a Gabriel-Roiter inclusion $M\left(\mathbf{x}, k_{0}\right) \rightarrow M\left(\mathbf{y}, k_{0}\right)$.

$\left(\mathbf{3}^{\prime \prime}\right)$ If there is a Gabriel-Roiter inclusion $M(\mathbf{x}, \mathbb{Q}) \rightarrow M(\mathbf{y}, \mathbb{Q})$, then there is a Gabriel-Roiter inclusion $M\left(\mathbf{x}, \mathbb{F}_{p}\right) \rightarrow M\left(\mathbf{y}, \mathbb{F}_{p}\right)$ for almost all prime numbers $p$. 
But this means the following:

(5) If there is a Gabriel-Roiter inclusion $M(\mathbf{x}, k) \rightarrow M(\mathbf{y}, k)$, for some field $k$, then there is a Gabriel-Roiter inclusion $M\left(\mathbf{x}, k^{\prime}\right) \rightarrow M\left(\mathbf{y}, k^{\prime}\right)$ for any field $k^{\prime}$.

Remark. We have shown that the existence of a Gabriel-Roiter inclusion $M(\mathbf{x}, k) \rightarrow M(\mathbf{y}, k)$ depends only on $\mathbf{x}, \mathbf{y}$, but not on $k$. If we consider arbitrary inclusions, the corresponding statement is not true. The typical example is furnished by the $D_{4}$-quiver with factorspace orientation, say with vertices $1,2,3,4$, where $1,2,3$ are sinks and 4 is a source. Let $X=P(4)$ (with dimension vector $\mathbf{x}=(1,1,1,1)$ ) and let $Y$ be indecomposable with dimension vector $\mathbf{y}=(1,1,1,2)$. Then, for $|k| \geq 3$, the module $M(\mathbf{x}, k)$ can be embedded into $M(\mathbf{y}, k)$. But for $k=\mathbb{F}_{2}$, there is no such embedding (the corresponding Hall polynomial is $\phi_{\mathbf{z}, \mathbf{x}}^{\mathbf{y}}(T)=T-2$ ).

\section{§6. Examples}

For the Bo Chen Theorem, all the assumptions on $\Lambda$ are necessary, as the following examples show:

(1) The algebra $\Lambda=k[T] / T^{2}$ is a representation-finite split $k$-algebra. The embedding of the simple $\Lambda$-module $S$ into the regular representation $P={ }_{\Lambda} \Lambda$ is a Gabriel-Roiter inclusion, but $\operatorname{Hom}(S, P / S) \neq 0$.

(2) If $\Lambda$ is the path algebra of a tame quiver, and $H$ is a simple homogeneous $\Lambda$-module, then there is an indecomposable $\Lambda$-module $H[2]$ with $H$ as a submodule such that $H[2] / H$ is isomorphic to $H$. One can show that $H$ is a Gabriel-Roiter submodule of $H[2]$ and $\operatorname{Hom}(H, H[2] / H) \neq 0$.

(3) In both cases (1) and (2), the algebra $\Lambda$ was not representationdirected. Let us consider now a representation-directed algebra which is not $k$-split, namely $\Lambda=\left[\begin{array}{ll}k & K \\ 0 & K\end{array}\right]$, with $k \subset K$ a field extension of degree 2. Note that $\Lambda$ is a hereditary algebra of Dynkin type $B_{2}$. We consider left $\Lambda$-modules. There are two indecomposable projective modules $P_{1}$ and $P_{2}$, with $P_{1}$ of length 1 and $P_{2}$ of length 3 . Any embedding $f: P_{1} \rightarrow P_{2}$ has as image a Gabriel-Roiter submodule of $P_{2}$, but $\operatorname{Hom}\left(P_{2}, \operatorname{Cok}(f)\right) \neq 0$. The corresponding Hall polynomial for $\mathbf{x}=\operatorname{dim} P_{1}, \mathbf{y}=\operatorname{dim} P_{2}, \mathbf{z}=$ $\operatorname{dim} \operatorname{Cok}(f)$ is

$$
\phi_{\mathbf{z x}}^{\mathbf{y}}(T)=T+1
$$

(in terms of the numbers $h, s, e, r$ : we have $h=2, s=0$ and $e=1, r=0$ ). 


\section{§7. Representation-finite algebras}

What are the building blocks of a length category? The obvious first (but naive) answer will be: take the simple objects and reconstruct any object along a composition series. However it turns out that this is a quite difficult procedure - just observe that most objects will have numerous and very different composition series! In case we deal with a hereditary length category, at least the exceptional objects can be reconstructed very well using the Schofield sequences. The following considerations seem to suggest that in general one should look at Gabriel-Roiter inclusions (and inductively Gabriel-Roiter filtrations).

Consider a representation-finite split $k$-algebra $\Lambda$ and an indecomposable $\Lambda$-module $Y$. We are interested in concise ways for writing $Y$ as an extension of smaller modules. We can assume that $Y$ is faithful (and not simple). By a result of Bongartz [B], this implies that $\Lambda$ has a simply connected universal covering $\widetilde{\Lambda}$ with a dense covering functor $\pi: \bmod \widetilde{\Lambda} \rightarrow \bmod \Lambda$. Here, $\widetilde{\Lambda}$ is a split $k$-algebra, not necessarily with 1 , but with sufficiently many idempotents $e_{i}$ (this means that $\widetilde{\Lambda}=\bigoplus_{i, j} e_{i} \widetilde{\Lambda} e_{j}$ ) and $\widetilde{\Lambda}$ is locally bounded (all the ideals $\widetilde{\Lambda} e_{i} \widetilde{\Lambda}$ are finite-dimensional).

If we denote by $\widetilde{Y}$ an indecomposable object in $\bmod \widetilde{\Lambda}$ with $\pi(\widetilde{Y})=Y$, and by $I$ its annihilator, then $\Lambda_{Y}=\widetilde{\Lambda} / I$ is a representation-directed split $k$-algebra and properties of $Y$ can be studied by looking at the $\Lambda_{Y}$-module $\tilde{Y}$.

The covering terminology used by Bongartz and Gabriel may be reformulated as follows: the algebra $\Lambda$ can be $G$-graded with $G$ a (usually non-abelian) free group, called the Galois group of the covering, and we may identify $\bmod \widetilde{\Lambda}$ with the category of $G$-graded $\Lambda$-modules, so that $\pi$ is just the forgetful functor. Of importance is that $\pi$ is a dense functor: this means that any $\Lambda$-module can be $G$-graded. Given any $G$-graded $\Lambda$ module $M$, and $g \in G$, we denote by $g M$ the $G$-graded $\Lambda$-module with grading shifted by $g$. Of course, $\pi(g M)=\pi(M)$ and there is the following "covering property": Let $M, N$ be $G$-graded $\Lambda$-modules, then $\pi$ yields a bijection

$$
\bigoplus_{g \in G} \operatorname{Hom}_{\widetilde{\Lambda}}(g M, N) \stackrel{\sim}{\longrightarrow} \operatorname{Hom}_{\Lambda}(\pi(M), \pi(N)) .
$$

Assume now that $\Lambda$ and $\Lambda^{\prime}$ are locally bounded split $k$-algebras with sufficiently many idempotents. 
Proposition 4. Let $\pi: \bmod \Lambda^{\prime} \rightarrow \bmod \Lambda$ be a dense covering functor with Galois group $G$. Let $X, Y$ be indecomposable in $\bmod \Lambda^{\prime}$.

(a) If $u: X \rightarrow Y$ is a monomorphism in $\bmod \Lambda^{\prime}$, then $\pi(u): \pi(X) \rightarrow$ $\pi(Y)$ is a monomorphism in $\bmod \Lambda$.

(b) Let $v: \pi(X) \rightarrow \pi(Y)$ be a monomorphism in $\bmod \Lambda$ with image a Gabriel-Roiter submodule of $\pi(Y)$. Then there exists a monomorphism $v^{\prime}: g X \rightarrow Y$ for some $g \in G$ such that $g X$ is a Gabriel-Roiter submodule of $Y$.

(c) $\mu(Y)=\mu(\pi(Y))$.

Proof. Assertion (a) is obvious and this yields the inequality $\mu(Y) \leq$ $\mu(\pi(Y))$ in $(\mathrm{c})$.

The converse inequality of (c) and statement (b) will be shown by induction on the length of $Y$. Using the bijection

$$
\bigoplus_{g \in G} \operatorname{Hom}_{\widetilde{\Lambda}}(g X, Y) \longrightarrow \operatorname{Hom}_{\Lambda}(\pi(X), \pi(Y)),
$$

the map $v: \pi(X) \rightarrow \pi(Y)$ can be written as $v=\sum_{g \in G} \pi\left(v_{g}\right)$ with $v_{g}: g X \rightarrow Y$ (and only finitely many of these maps $f_{g}$ being non-zero). According to Proposition 1, at least one of the maps $\pi\left(v_{g}\right)$ has to be injective, thus we obtain a monomorphism $v_{g}: g X \rightarrow Y$.

By induction, we may assume that $\mu(X)=\mu(\pi(X))$, and since $\pi(g X)=$ $\pi(X)$, also $\mu(g X)=\mu(\pi(X))$. Let $n=|Y|=|\pi(Y)|$. Now, according to the definition of $\mu(Y)$,

$$
\mu(\pi(Y))=\mu(\pi(X))+2^{-n}=\mu(g X)+2^{-n} \leq \mu(Y) .
$$

But since we know already that $\mu(Y) \leq \mu(\pi(Y))$, we have equality and therefore (c) holds. We also see that $\mu(g X)+2^{-n}=\mu(Y)$, and thus the image of $v^{\prime}=v_{g}$ is a Gabriel-Roiter submodule of $Y$. This completes the proof.

In particular, we see the following: Let $\Lambda$ be a representation-finite split $k$-algebra and $Y$ a faithful and indecomposable $\Lambda$-module. Let $\pi$ : $\bmod \widetilde{\Lambda} \rightarrow \bmod \Lambda$ the universal covering and $\widetilde{Y}$ an indecomposable object in $\bmod \widetilde{\Lambda}$ with $\pi(\widetilde{Y})=Y$. Then

$$
\mu(\tilde{Y})=\mu(Y),
$$


and the Gabriel-Roiter filtrations of $\widetilde{Y}$ correspond to the Gabriel-Roiter filtrations of $Y$. Here $\tilde{Y}$ may be considered as a $\Lambda_{Y}$-module, where $\Lambda_{Y}=$ $\widetilde{\Lambda} / I$ with $I$ the annihilator of $\widetilde{Y}$. The algebra $\Lambda_{Y}$ is a representation-directed split $k$-algebra, so that the Bo Chen Theorem applies.

Remark 1. If $\Lambda$ is a representation-directed and split $k$-algebra, then we know that for any Gabriel-Roiter inclusion $X \subset Y$ we have $\operatorname{dim}_{k}$ $\operatorname{Hom}(X, Y)=1$, thus in particular $X$ is the only submodule of $Y$ which is isomorphic to $X$. This is no longer true for representation-finite split $k$-algebras. A typical example is given by the quiver $\widetilde{A}_{2,1}$ with zero relation

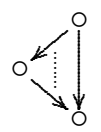

and the indecomposable representation $Y$ of dimension 4. The GabrielRoiter measure of $Y$ is given by $I(Y)=\{1,2,4\}$. There is a family of Gabriel-Roiter submodules $X$ of $Y$ indexed by the affine line $\mathbb{A}^{1}$ (and $\operatorname{dim}_{k} \operatorname{Hom}(X, Y)=2$ ). Covering theory considers instead of this cycle a quiver of type $A_{\infty}^{\infty}$ (with infinitely many zero relations):

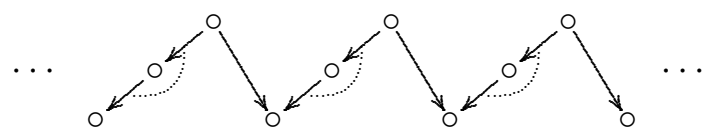

The corresponding modules $\widetilde{Y}$ are the only indecomposable representation of dimension 4 and any such module has, in contrast to $Y$, a unique GabrielRoiter submodule. In terms of graded algebras and graded modules, we consider $Y$ as being endowed with a grading such that $Y$ is written as the direct sum of four 1-dimensional subspaces.

Remark 2. Let us stress that for a Galois covering $\pi: \bmod \Lambda^{\prime} \rightarrow$ $\bmod \Lambda$ and indecomposable $\Lambda^{\prime}$-modules $X, Y$, the existence of an embedding $\pi(X) \rightarrow \pi(Y)$ usually does not imply the existence of an embed$\operatorname{ding} X \rightarrow Y$. A typical example is provided by the universal covering $\pi: \bmod \Lambda^{\prime} \rightarrow \bmod \Lambda$ with Galois group $G=\mathbb{Z}:$

$\Lambda^{\prime}$

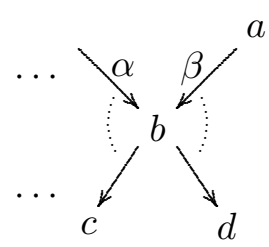

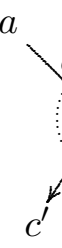

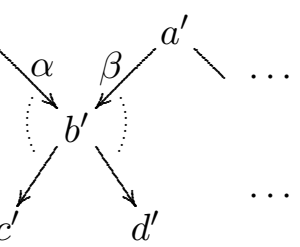

$\Lambda$

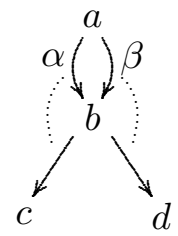


For a vertex $x$ of $\Lambda$, we denote by $P_{\Lambda}(x)$ the corresponding indecomposable projective module, and similarly for vertices of $\Lambda^{\prime}$. There is an embedding $u$ of $P_{\Lambda}(b)$ into $P_{\Lambda}(a)$. However, there is no monomorphism from $P_{\Lambda^{\prime}}\left(b^{\prime \prime}\right)$ into $P_{\Lambda^{\prime}}(a)$ for any vertex $b^{\prime \prime}$ in the $G$-orbit of $b$, but there are non-zero maps $P_{\Lambda^{\prime}}(b) \rightarrow P_{\Lambda^{\prime}}(a)$ and $P_{\Lambda^{\prime}}\left(b^{\prime}\right) \rightarrow P_{\Lambda^{\prime}}(a)$, neither one being a monomorphism, such that the images under $\pi$ add up to the monomorphism $u$ (in particular, $u$ belongs to the additive closure of $\left.\operatorname{Sing}\left(P_{\Lambda}(b), P_{\Lambda}(a)\right)\right)$.

\section{REFERENCES}

[B] K. Bongartz, Indecomposables are standard, Comment. Math. Helv., 60 (1985), 400-410.

[BG] K. Bongartz and P. Gabriel, Covering spaces in representation theory, Invent. Math., 65 (1982), 331-378.

[C] B. Chen, The Gabriel-Roiter measure for representation-finite hereditary algebras, Dissertation, Bielefeld 2006.

[CB] W. Crawley-Boevey, Rigid integral representations of quivers, Can. Math. Soc. Conf. Proc. 18 (1996), pp. 155-163.

[G] P. Gabriel, Indecomposable representations II, Symposia Mat. Inst. Naz. Alta Mat., 11 (1973), 81-104. MR 49:5132.

[L] G. Lusztig, Canonical bases arising from quantized enveloping algebras, J. Amer. Math. Soc., 3 (1990), 447-498.

[M] I. G. Macdonald, Symmetric functions and Hall polynomials, second edition, Oxford Math. Mon., 1995.

[R1] C. M. Ringel, Tame algebras and integral quadratic forms, Springer LNM 1099, 1984 .

[R2] C. M. Ringel, Hall algebras, Topics in Algebra, Banach Center Publ. 26 (1990), pp. $433-447$.

[R3] C. M. Ringel, Hall polynomials for the representation-finite hereditary algebras, Adv. Math., 84 (1990), 137-178.

[R4] C. M. Ringel, Exceptional objects in hereditary categories, Proceedings Constantza Conference, An. St. Univ. Ovidius Constantza Vol. 4 (1996), f. 2, 150-158.

[R5] C. M. Ringel, The braid group action on the set of exceptional sequences of a hereditary algebra, Abelian Group Theory and Related Topics, Contemp. Math. 171 (1994), pp. 339-352.

[R6] C. M. Ringel, The Gabriel-Roiter measure, Bull. Sci. math., 129 (2005), 726-748.

[R7] C. M. Ringel, Foundation of the Representation Theory of Artin Algebras, Using the Gabriel-Roiter Measure, Proceedings ICRA 11, Queretaro 2004, Contemporary Math. 406, Amer. Math. Soc. (2006), pp. 105-135.

[S] A. Schofield, The internal structure of real Schur representations, preprint, 46-64. 
Fakultät für Mathematik

Universität Bielefeld

POBox 100131

D-33501 Bielefeld

Germany

ringel@math.uni-bielefeld.de 